\section{Experimental and Numerical Study of Two Dimensional Flow of Bubble Separation over the Leading of Thickness Plate}

\author{
Herman Sasongkd ${ }^{\circ}$, Abdul Haris Irfani \\ Department of Mechanical Engineering, Institut Teknologi Sepuluh Nopember, Surabaya 60111, Indonesia \\ Received: 12 March 2020, Revised: 13 September 2020, Accepted: 14 September 2020
}

\begin{abstract}
Various design modifications made by transportation equipment companies aim to increase the efficiency of fuel consumption. One of them is by reducing the drag force. This can be done by controlling passive turbulent boundary layers. The addition of rounded shape on the leading edge which is correlated with the length of the plane is one way of controlling the passive turbulent boundary layer which can accelerate the transition from the laminar boundary layer to the turbulent boundary layer. Therefore, this study aims to determine the effect of the Reynolds number and flow support length on the flow characteristics so that the downstream separation can be delayed. This research was conducted by experimental and numerical methods. The Reynolds number used is $R e_{t}=5.08 \times 10^{4}$ and $R e_{t}=8.46 \times 10^{4}$. The test part of this research is the rounded leading edge of thick plate with a plane length of $c / t=6.5$ and $c / t=10$. The amount of rounded on the leading edge, the height of the test model and the length of the trailing edge are $10 \mathrm{~mm}, 100 \mathrm{~mm}$, and $300 \mathrm{~mm}$, respectively. The parameters varied in this study were the flow bearing field length $(c / t)$ and the Reynolds number $\left(R e_{t}\right)$. Two-dimensional, steady numerical simulation was carried out using ANSYS Fluent software. The turbulence model used is $k-k l-\omega$. The results obtained in this study are the longer the flow bearing plane and the greater the Reynolds number can delay the separation on the upper side of the circular front edge of the thick plate. The optimal length of the separation delay time is $c / t=10$ with $R e_{t}=8.46 \times 10^{4}$. The separation bubble profile at $c / t=10$ with $R e_{t}=8.46 \times 10^{4}$ has length $(x / c)=0.129$, thickness $(y / t)=0.1363$, and angle $(\Theta)=30.3^{\circ}$ with the form factor $(H)$ at point $O$ 1.424. Overall the optimal variation is at $c / t=10$ with $R e_{t}=8.46 \times 10^{4}$ where the point of separation occurs when $x / c=0.945$.
\end{abstract}

Keywords: Bubble separation, rounded leading edge, Reynolds number, plane length

\section{Introduction}

Various design modifications made by transportation equipment companies aim to increase the efficiency of fuel consumption. One of them is by reducing the value of the drag force. Research on reducing the value of the drag force is carried out in various ways, one of which is the turbulent boundary layer control method. There are two methods of turbulent boundary layer control, namely passive turbulent boundary layer control and active turbulent boundary layer control. In passive turbulent boundary layer control, control is done by adding a permanent disturbance attached to the object. Meanwhile, the active boundary-layer control is more flexible because it adapts to the desired needs [1].

One of the objectives of the passive turbulent boundary layer control is to attempt a bypass transition of the laminar to turbulent boundary layer by forming a bubble separation. The formation of bubble separation begins with flow separation which then reattach. The bubble separation process, which is immediately followed by the formation of a turbulent boundary layer, is used to delay and even eliminate permanent flow separation in the downstream area. Lamballais et al [2] conducted a study on bubble separation using numerical methods on semi-thick plates. Lamballais et al varied the curvature at the leading edge $(\eta)$, namely $0.125,0.25,0.5$, and 1 . In addition, numerical methods were carried out using 2-dimensional and 3-dimensional objects for each size of curvature, as well as variations in the excitation inlet $\left(u_{\text {inflow }}^{\prime}\right)$ of 0 and $0.1 \%$ of $U_{\infty}$. This study used the numerical direct numerical Simulation (DNS) method, the thickness of the half plate measuring $20.25 H$ to the x-axis, $16 H$ to the y-axis, and $6 H$ to the z-axis, and the Reynolds number 2000 . The study showed that in 3-dimensional objects, the separation of bubbles is more dominant than in 2-dimensional objects. In 3-dimensional objects, the formed bubble separation becomes more unstable as the curvature increases. Whereas for 2-dimensional objects the results are not in accordance with the theory, especially in the reverse flow which is formed compared to the results of 3-dimensional objects. The length of recirculation in the bubble separation area, the bubble separation height, and the bubble

*Corresponding author. Email: herman@me.its.ac.id 
separation angle are also observed. In a 3D object, the length of the recirculation increases by $+60 \%$ when $\eta=1$, and $+7 \%$ when $\eta=0.125$. Whereas in a 2 -dimensional object the length of the recirculation increases by $+23 \%$ when $\eta=1$, and is no longer affected by the inflow velocity when $\eta \leq 0.5$. Meanwhile, the height of the bubble separation decreases as the curvature at the front end in creases. At $\eta=1$, the bubble separation height is about $+42 \%$ and at $\eta=0.125$ the bubble separation height is about $+4 \%$ for a 3D object. The change in bubble size does not affect the angle at which the bubble separation is formed.

Niu et al [3] studied drag and bubble separation using numerical methods on high-speed trains with different nose lengths. Niu et al varied the length of the train's nose to be short and long without an angle of attack. Niu et al using Fluent 18.1 software with input including turbulent model is SST $k-\omega$, SIMPLE pressure coupling and speed with second order downwind, unstable flow conditions, and $50 \mathrm{~m} / \mathrm{s}$ inlet speed. The results showed that the effect of the length of the train nose from $8 \mathrm{~m}$ to $12 \mathrm{~m}$ can reduce drag by $30.53 \%$ and increase lift by $87.98 \%$. In addition, the boundary layer profile is influenced by the length of the nose train where the bubble separation is more stable in the longer nose train.

Based on the previous studies, the opportunity to reattach to form a bubble separation due to the influence of dynamic force (Reynolds number) which correlates with the flow bearing plane length and the shape of a certain leading edge has hardly been studied. In this case, the field length of the flow bearing means the distance between two potential front and rear separation points. The initial hypothesis was that elongation of the plane length could facilitate re-fitting of the separated streams on the leading edge, which affected the size of the separation bubbles formed and could delay the separation of the flow in the downstream area. Then a study was conducted to optimize the delay in flow separation in the downstream area. This research was conducted with an experimental method which was validated by numerical methods.

\section{Method}

In accordance with the research objectives, testing is carried out through experimental and numerical methods with a specific test model. This experiment will be carried out in a wind tunnel at the Mechanical Engineering Department Workshop, FTIRS-ITS. The model used is a thick plate round leading edge with a round leading edge with a certain radius and an oval trailing edge. Figure 1 shows the measurement parameters used in this study.

The test model has a height of $100 \mathrm{~mm}$ with a round tip of $10 \mathrm{~mm}$ and a width of $660 \mathrm{~mm}$. The length of the flow support which is a variation in this study has a length of $350 \mathrm{~mm}$ and $700 \mathrm{~mm}$ with a trailing edge in the form of a quarter ellipse that has a fixed length of $300 \mathrm{~mm}$, as shown in Figure 2. The two lengths of the flow bearing plane will be observed at two different Reynolds numbers, namely at $R e_{t}=5.08 \times 10^{4}$ and $R e_{t}=8.46 \times 10^{4}$. This study has the test configuration shown in Table 1 .
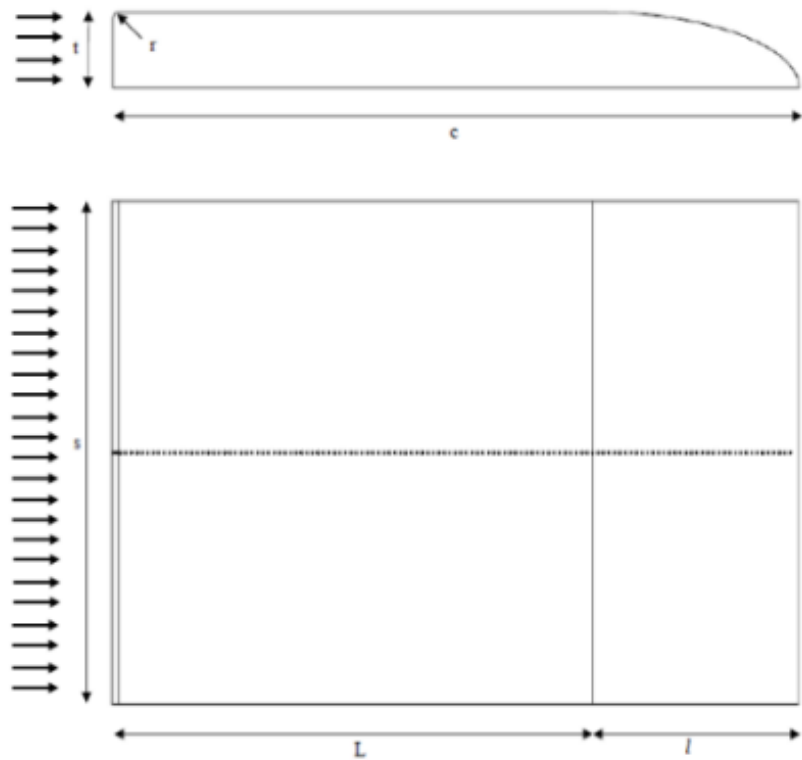

With;

$r \quad=$ the radius of the leading edge

$L \quad=$ field length of the flow bearing

$l=$ back edge length

$c \quad=$ chord length

$s \quad=$ length / width of the body

$t \quad=$ height

Figure 1. Measurement parameters 


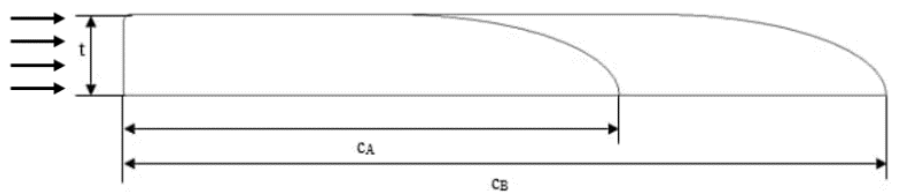

Figure 2. Test configuration

Table 1. Test configuration

\begin{tabular}{ccc}
\hline Configuration & $c / t$ & $R e$ \\
\hline A & 6.5 & $5.08 \times 10^{4}$ \\
B & 6.5 & $8.46 \times 10^{4}$ \\
C & 10 & $5.08 \times 10^{4}$ \\
D & 10 & $8.46 \times 10^{4}$ \\
\hline
\end{tabular}

\subsection{Experimental Method}

The wind tunnel is a test area for specimens with a test cross section in the form of a beam with a length of $1780 \mathrm{~mm}$, a width of $660 \mathrm{~mm}$ and a height of $660 \mathrm{~mm}$ and is capable of operating at a maximum speed of $21 \mathrm{~m} / \mathrm{s}$. Figure 3 shows the wind tunnel scheme used in this study.

Wall pressure tap is used to measure the static pressure of fluid flow which is mounted on the upper side of the surface of the specimen as shown in Figure 1. In the variation of $c / t=6.5$ there are 156 wall pressure taps and at $c / t=10$ there are 223 wall pressure taps. In this study, what is measured is static pressure and stagnation pressure. Several supporting tools were used during the experiment, including: pitot static tube, pressure transducer, data acquisition, manometer, and thermometer.

\subsection{Numerical Method}

The numerical method was carried out using ANSYS (Fluent) software and in this study a two-dimensional model was used. The selected and approached mesh shape of the experimental results is rectangular on all parts of the wall surface. The test model net is shown in Figure 4. Boundary Condition is the determination of parameters and limits on flow, namely by providing load speed, pressure and turbulence boundary conditions at the inlet, outlet and wall conditions. In the test model for the numerical method it is assumed that the length of 1 chord, the distance of the inlet and leading edge is 1 chord, and the distance of the trailing edge and outlet is 4 chords. The inlet boundary conditions are given according to the variation of velocity, namely $7.5 \mathrm{~m} / \mathrm{s}$ and $12.5 \mathrm{~m} / \mathrm{s}$, while at the outlet there is a pressure outlet of $0 \mathrm{~Pa}$. In this study the turbulence model used is $k-k l-\omega$. The inlet turbulence intensity was set to $4.1298 \%$ and the turbulence length scale was $0.0462 \mathrm{~m}$. The simulation domain and boundary conditions are shown in Figure 5

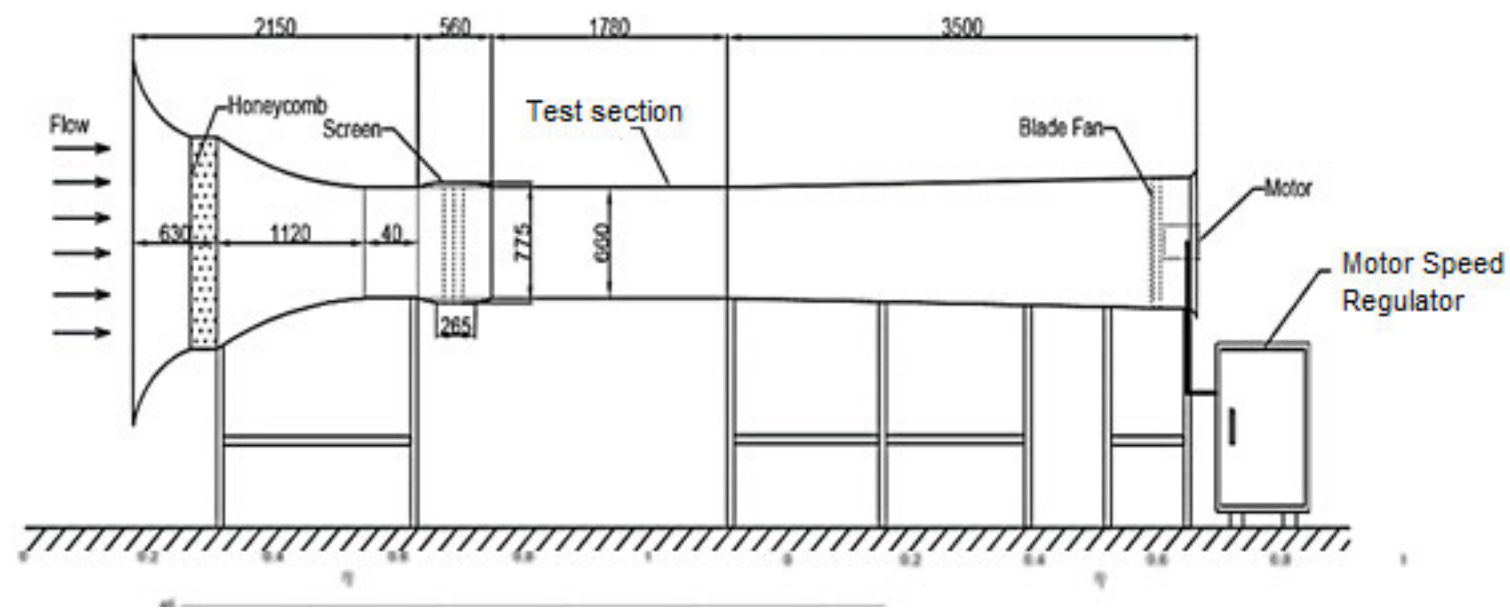

Figure 3. Schematic of the wind tunnel used 


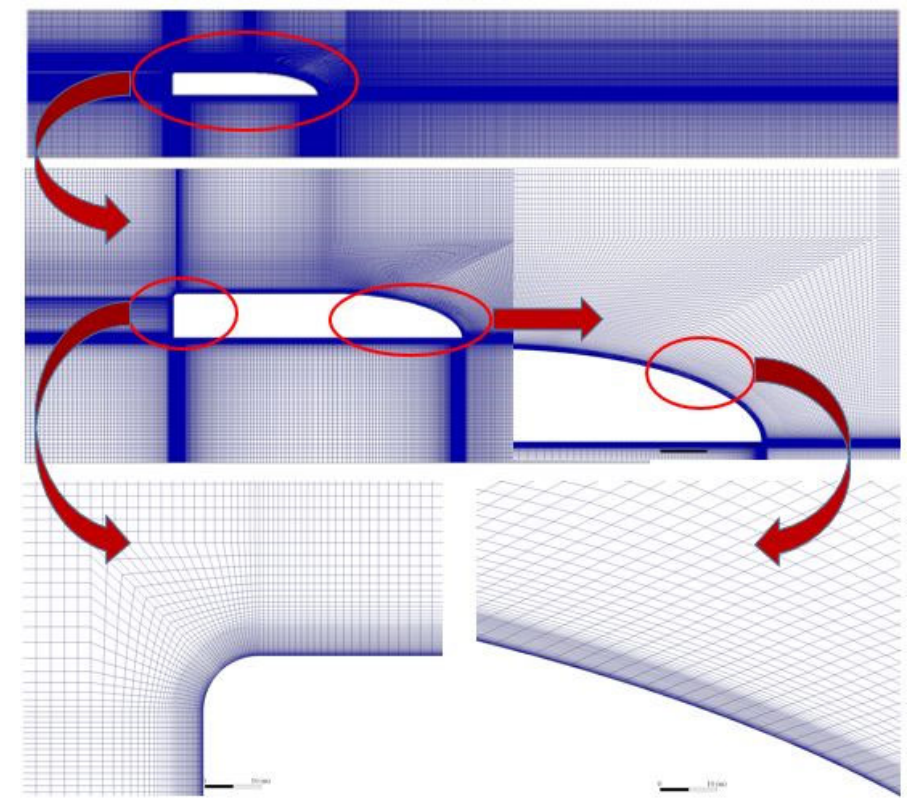

Figure 4. The test model for the meshing model at $c / t=6.5$

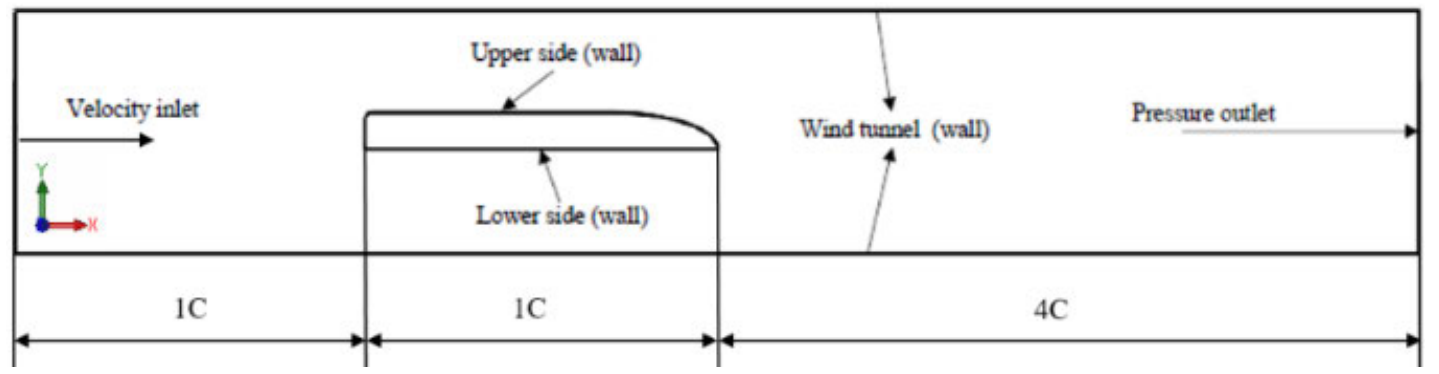

Figure 5. Simulation domains and boundary conditions

\subsection{Data Retrieval}

The experimental results taken are the static pressure value at the top of the test model which is then converted into a pressure coefficient distribution graph. Meanwhile, from the numerical method, the results of flow visualization include velocity contours and static pressure contours, streamlined displays, and velocity vector displays. In addition, quantitative data taken from numerical methods include the bubble separation profile, the length of the massive separation, the thickness of the boundary layer, the velocity profile and the form factor at point $O 300 \mathrm{~mm}$ as shown in Figure 6 .

\section{Results and Discussion}

\subsection{Data Validation}

Validation was carried out by comparing the value of the pressure coefficient on the upper side and the velocity profile at variation $c / t=6.5$ with $R e_{t}=5.08 \times 10^{4}$. In numerical methods, viscous models $k-k l-\omega$ and $S S T$ $k-\omega$ were used to find corresponding experimental results. The graph comparison of the pressure coefficient between the experimental results and the numerical results with $k-k l-\omega$ is shown in Figure 77. While the graph of the pressure coefficient comparison between the experimental results and the numerical results using $S S T k-\omega$ is shown in Figure 8 .

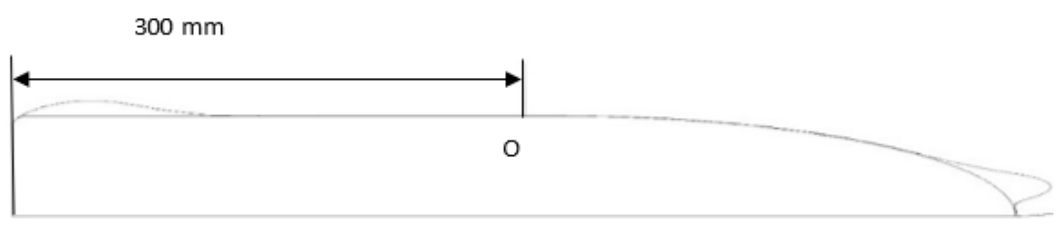

Figure 6. Velocity profile point distance and form factor 


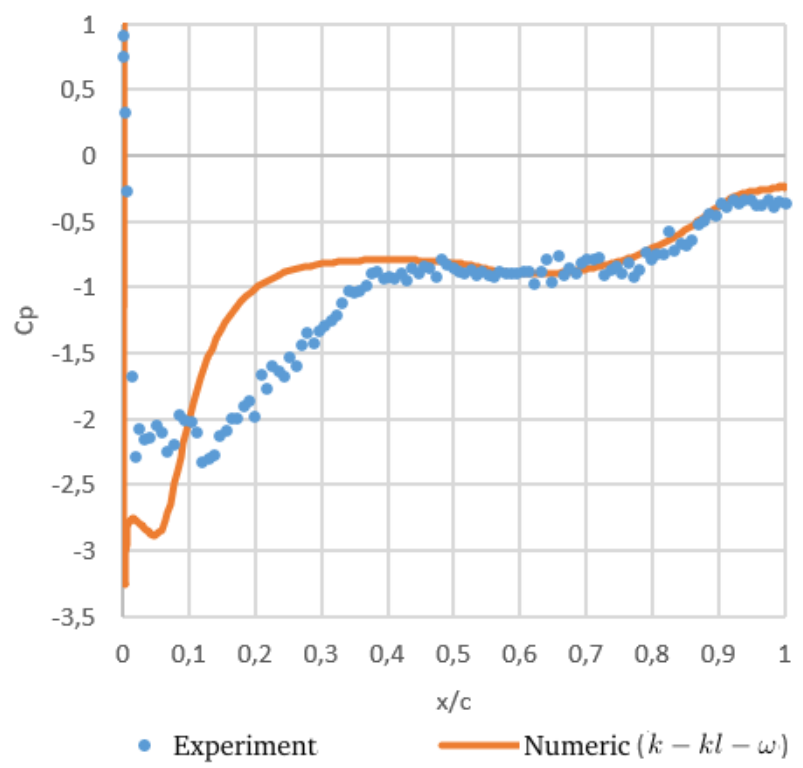

Figure 7. Experimental validation and numerical pressure coefficient $(k-k l-\omega)$ for $c / t=6.5$

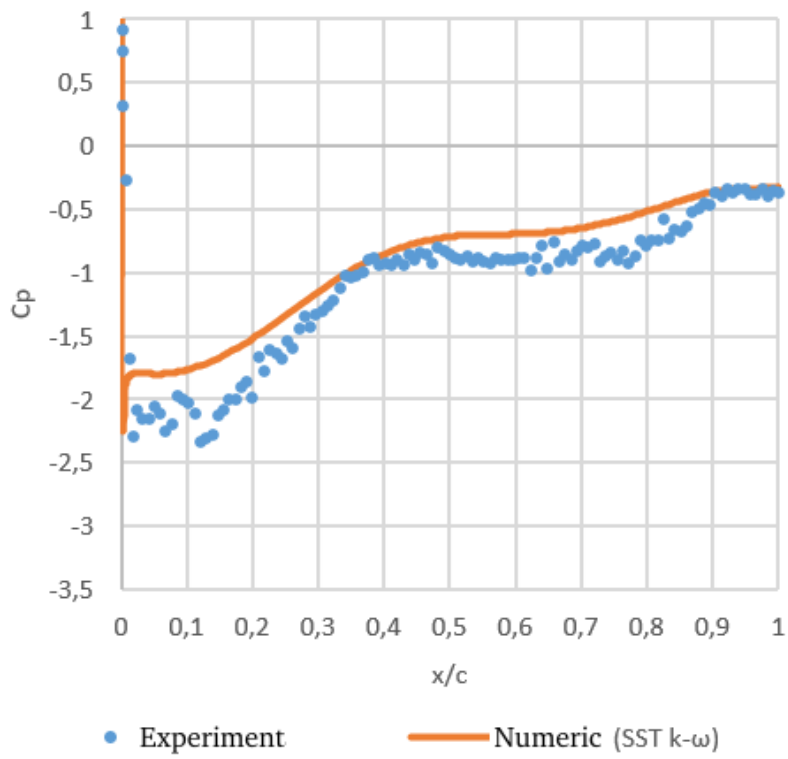

Figure 8. Validation of experimental results and numerical $C p(S S T k-\omega)$ for $c / t=6.5$

Based on Figures 7 and gambar8.png, it can be seen that the pressure coefficient $(C p)$ of the $x / c$ function numerical results with $k-k l-\omega$ has the same graph pattern as the experimental results. Where the flow experiences high acceleration on the leading side at the top of the side coming from the incoming side of the thick round plate with the $C p$ value decreases sharply because the flow passes through the convergence flow pipe. Then the $C p$ value has increased which indicates separation. In the graph of experimental and numerical results with $k-k l-\omega$ there are discontinuities. This is indicated by a slight decrease in the $C p$ value which then rises back to the re-attachment point. These results indicate that according to the existing theory, the statistical pressure tends to be constant and then rises again in the bubble separation. At this time there is a flow transition from the laminar boundary layer to the turbulent boundary layer. After passing the re-attachment point, the value of $C p$ is relatively constant. While the numerical results with $S S T$ $k-\omega$ differ from the experimental results, where there is no discontinuity in the bubble separation area.

To determine the viscous model used in the numerical method, validation is required in the form of a velocity profile. The graph of the numerical result velocity profile comparison at point $O$ between the $k-k l-\omega$ viscous models and $S S T k-\omega$ is shown in Figure 9 . 


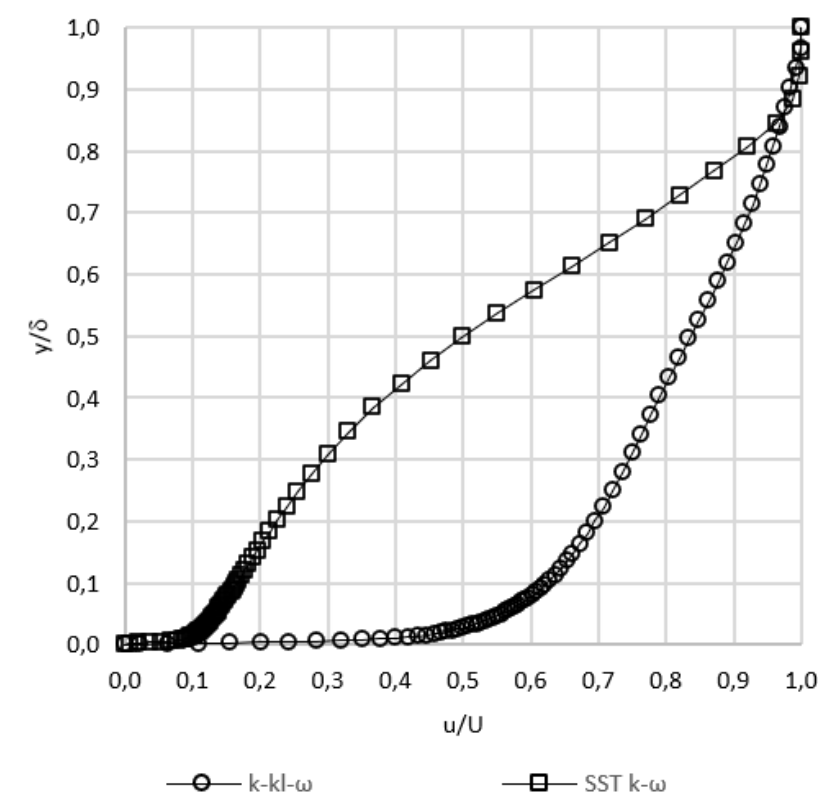

Figure 9. Validation of the numerical result velocity profile between $k-k l-\omega$ and $S S T k-\omega$

Based on Figure 9, it can be seen that the velocity profile of the $u / U$ and $y / \delta$ functions from the numerical results with the $k-k l-\omega$ viscous model has a velocity profile shape in accordance with the theory. Where the near-wall flow velocity profile has greater momentum which is used to counter shear stress and adverse stress. Meanwhile, the numerical results with the $k-\omega$ viscous SST model show a different velocity profile from the theory. Where the near-wall flow velocity profile has smaller momentum. At the point $x=0.3 \mathrm{~m}$, the flow crossing the upper side must have a relaxed turbulent boundary layer.

In general, numerical methods are used to simulate external flow, such as that of Niu et al.[3] using a thick model, namely $S S T k-\omega$. However, based on the validation results, the results of the $k-k l-\omega$ viscous model are more in accordance with the theory and experimental results. So that in this research the viscous model, $k-k l-\omega$, is used to simulate the flow across the leading edge in the form of a thick round plate.

\subsection{Grid Independence Analysis}

The independence of the grid is done by varying the amount of meshing on the round leading edge of the thick plate with the flow support length $c / t=6.5$ and $c / t=10$ at $R e_{t}=5.08 \times 10^{4}$. Meshing $\mathrm{A}$ is the most coarse meshing where at $c / t=6.5$ with the number of cells 167,784 , while at $c / t=10$ with the number of cells 213,304 . Whereas Meshing $E$ is the finest meshing where at $c / t=6.5$ with the number of cells 334,452 , while at $c / t=10$ with the number of cells 424,248 . From the iteration results, the value of $C p$ is obtained as in Table 2 for $c / t=6.5$ and in table 3 for $c / t=10$.

From Table 2 and 3 it can be seen that meshing $A$, $B, C$, and $\mathrm{D}$ experienced an increase in the number of cells by $\pm 10,000$, while meshing $E$ experienced a twofold increase in the number of cells from meshing $A$ Anderson [4]. Based on the iteration results, the $C p$ value for all types of meshing is almost the same (error $\leq 2 \%$ ). It is concluded that by increasing the number of cells, the change in the value of $C p$ is no longer significant so that grid independence is achieved. Furthermore, meshing variation $A$ is used to perform numerical solutions in Fluent modeling.

Table 2. Analysis of $C p$ independence grid at $x=50 \% C$ at $c / t=6.5$

\begin{tabular}{ccccc}
\hline Meshing & $\begin{array}{c}\text { Number } \\
\text { of cells }\end{array}$ & $\begin{array}{c}C p \\
50 \% C\end{array}$ & Error (\%) & $Y+$ \\
\hline A & 167,784 & -0.81862 & - & 3.01 \\
B & 176,584 & -0.81871 & 0.011 & 3.01 \\
C & 186,932 & -0.81912 & 0.061 & 3.01 \\
D & 196,986 & -0.82103 & 0.29 & 3.01 \\
E & 334,452 & -0.82953 & 1.33 & 3.009 \\
\hline
\end{tabular}

Table 3. Analysis of $C p$ independence grid at $x=50 \% C$ at $c / t=10$

\begin{tabular}{ccccc}
\hline Meshing & $\begin{array}{c}\text { Number } \\
\text { of cells }\end{array}$ & $\begin{array}{c}C p \\
50 \% C\end{array}$ & Error (\%) & $Y+$ \\
\hline A & 213,304 & -0.75192 & - & 3.001 \\
B & 221,664 & -0.7521 & 0.024 & 3.001 \\
C & 231,264 & -0.75224 & 0.043 & 2.999 \\
D & 241,384 & -0.75235 & 0.057 & 2.999 \\
E & 424,248 & -0.76152 & 1.28 & 2.998 \\
\hline
\end{tabular}




\subsection{Comparative Analysis of Flow Characteristics}

The numerical advantage is that it can identify changes in flow patterns passing through the test model. Changes in flow patterns can be displayed by visualizing velocity contours and pressure contours. In Figure 10, the contours of velocity and pressure at $c / t=6.5$ $R e_{t}=5.08 \times 10^{4}$ and $R e_{t}=8.46 \times 10^{4}$ are compared, which have almost the same flow visualization despite different ranges of values.

To explain the flow phenomenon that occurs around the test model, it can be explained qualitatively through streamlined visualization combined with velocity contours from the numerical results as shown in Figure 11

Based on Figure 11, the flow visualization is shown on the front edge of the rounded thickness of the plate $c / t=6.5$ with $R e_{t}=5.08 \times 10^{4}$ and $R e_{t}=8.46 \times 10^{4}$. In the flow visualization it can be seen that the stagnation point is not right in the middle of the model test because the top is rounded at the leading edge. This causes flow to accelerate at the leading edge and flow separation to occur after rounding. Then the flow momentum will increase as it passes through the convergence flow tube. This is indicated by the red contour on the upper edge of the thick leading edge of the leading edge round plate. After that the flow will pass through the divergence stream tube where the flow velocity will decrease which is indicated by the light blue contour. It can be seen that the flow forms a bubble separation marked by a reattachment point where the light blue contour turns green in the area near the test model at a certain distance from the leading edge.

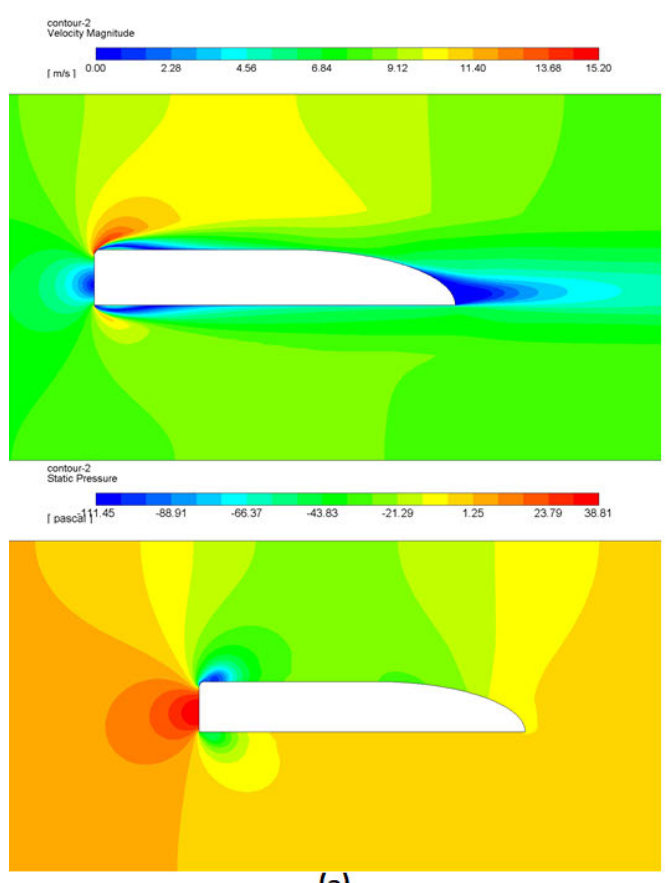

(a)

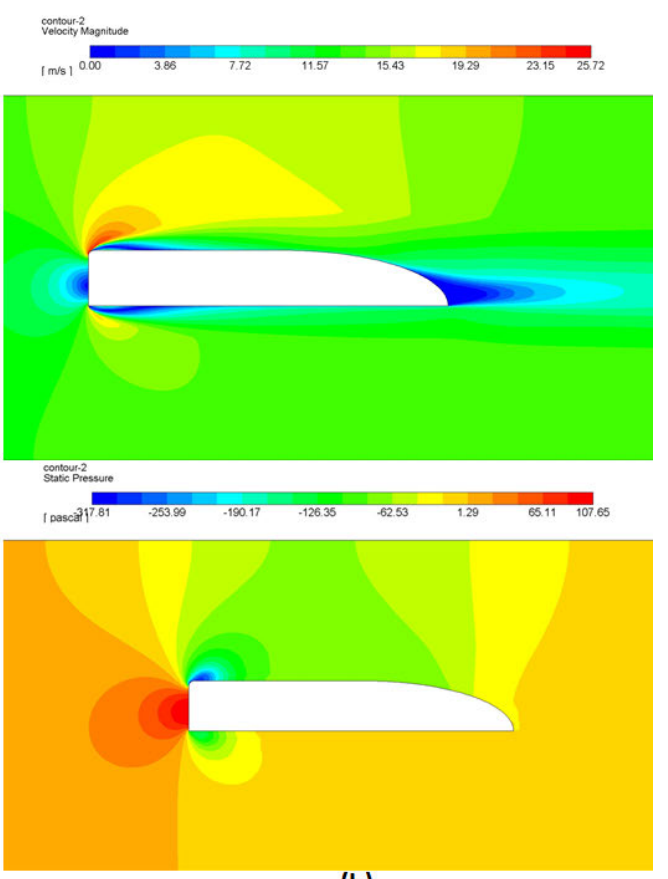

(b)

Figure 10. Comparison of velocity and pressure contours at $c / t=6.5$ with (a) $R e_{t}=5.08 \times 10^{4}$ and (b) $R e_{t}=8.46 \times 10^{4}$

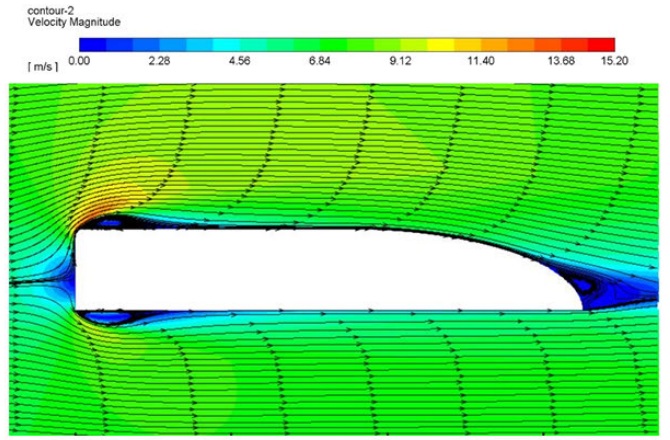

(a)

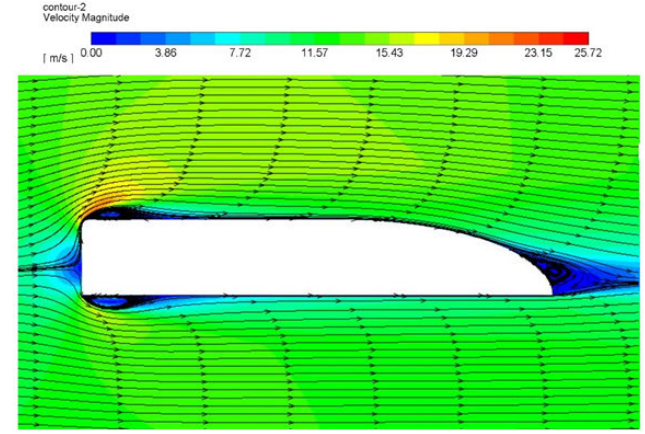

(b)

Figure 11. Streamline visualization at $c / t=6.5$ with (a) $R e_{t}=5.08 \times 10^{4}$ and (b) $R e_{t}=8.46 \times 10^{4}$ 
During reattachment, there is a transition from the laminar boundary layer to the turbulent boundary layer. Furthermore, the flow which has more momentum is directed towards the trailing edge. As it passes through the trailing edge, the flow undergoes massive separation. This occurs because the momentum possessed by the flow is no longer able to withstand the adverse stresses and shear stresses. The area behind the separation point is an area of very low velocity and there is a whirlpool. This area is called the wake area.

The pressure coefficient value from the numerical method is taken at the top of the test model. The data displayed in the graph of the pressure coefficient $(C p)$ against $x / c$ at $c / t=6.5$ with $R e_{t}=5.08 \times 10^{4}$ and $R e_{t}=8.46 \times 10^{4}$ are presented in Figure 12 .

Based on Figure 12, the comparison of the numerical results of the distribution of the pressure coefficient $(\mathrm{Cp})$ of the $x / c$ function at $c / t=6.5$ with $R e_{t}=5.08 \times 10^{4}$ and $R e_{t}=8.46 \times 10^{4}$. In this graph, it is known that the point of stagnation is has the highest $C p$ value, namely 1 , both $R e_{t}=5.08 \times 10^{4}$ and $R e_{t}=8.46 \times 10^{4}$ at $y / t=0.355$ when $x / c=0$. After that, it can be seen that the flow is experiencing high acceleration at the spearhead. On the upper side of the front edge, the thickness of the round plate is indicated by the $C p$ value which decreases sharply until it experiences separation when $x / c=0.003$ with a minimum $C p$ value of -3.27 for $R e_{t}=5.08 \times 10^{4}$ and at -3.33 for $R e_{t}=8.46 \times 10^{4}$. This is because the flow passes through the convergence flow tube so that the flow experiences a fluctuating acceleration. Then the $C p$ value has increased which indicates separation. The numerical result graph shows the discontinuities in the bubble separator area. This is marked by a slight decrease in the value of $C p$ which then rises back to the reattachment point, namely at $x / c=0.215$ for $R e_{t}=5.08 \times 10^{4}$ and at $x / c=0.205$ for $R e_{t}=8.46 \times 10^{4}$. pass through the divergence of the flow pipe so that the flow velocity is reduced. At this time there is a flow transition from the laminar boundary layer to the turbulent boundary layer. After passing the reattachment point, the $C p$ value is relatively constant. Then the flow will undergo massive separation because it is no longer able to withstand the surface shear stress and the adverse stress in the trailing edge area. The point of separation occurs at $x / c=0.885$ for $R e_{t}=5.08 \times 10^{4}$ and at $x / c=0.899$ for $R e_{t}=8.46 \times 10^{4}$. The greater the freestream flow velocity, the greater the flow momentum. Flow momentum is used to resist shear stress and adverse stress so that separation is delayed in the downstream area. The graph shows the same pattern of the $C p$ value on the upper side where at $R e_{t}=8.46 \times 10^{4}$ it is more effective to delay the separation in the downstream area.

To determine the effect of the flow bearing length and Reynolds number in increasing flow momentum, the comparative data of the velocity profile between $R e_{t}=5.08 \times 10^{4}$ and $R e_{t}=8.46 \times 10^{4}$ at point $O$ are presented as in Figure 13

Based on Figure 13 it can be seen that the velocity profiles of the $u / U$ and $y / \delta$ functions experience a slowdown due to adverse shear stress and stress. The greater the Reynolds number value, the greater the flow velocity near the surface of the test model so that the flow momentum increases. This flow momentum is used to counter shear stress and adverse stress so that the separation at the trailing edge is slower. In Figure 18 it can be seen that the test object with a variation of $R e_{t}=8.46 \times 10^{4}$ has a velocity closer to the surface that is greater than that of $R e_{t}=5.08 \times 10^{4}$.

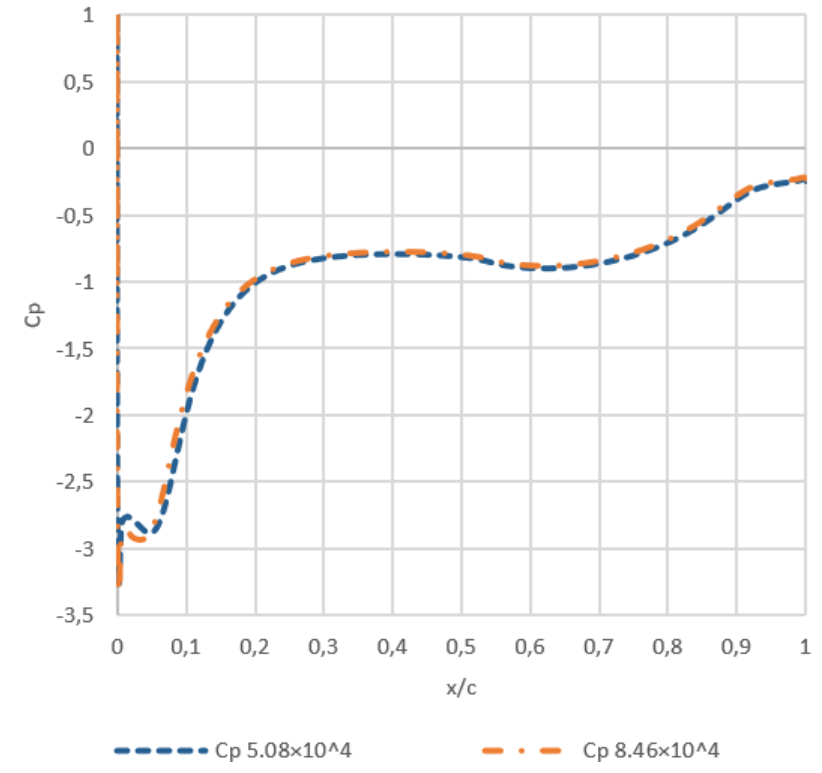

Figure 12. Comparison of $\mathrm{Cp}$ with $c / t=6.5$ with at $R e_{t}=5.08 \times 10^{4}$ and $R e_{t}=8.46 \times 10^{4}$ 


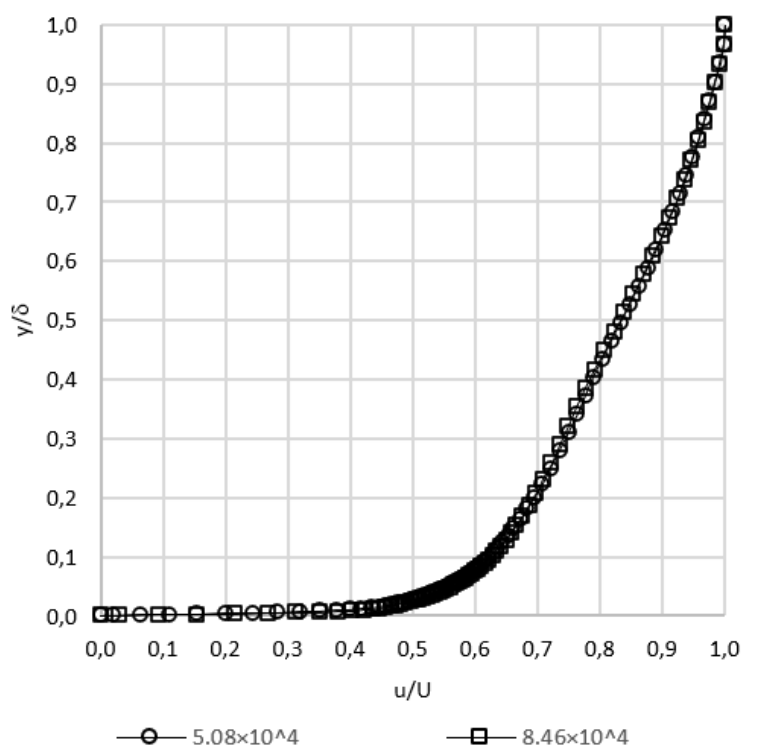

Figure 13. Velocity profile at the upper side point $O$

Apart from the velocity profile, to determine the effect of the flow bearing length and the Reynolds number in increasing the flow momentum can be seen from the turbulent kinetic energy graph. The plot comparison of the turbulent kinetic energy at point $O$ between $R e_{t}=5.08 \times 10^{4}$ and $R e_{t}=8.46 \times 10^{4}$ is shown in Figure 14

Figure 14 shows that the energy kinetic turbulence is a function of $k / U^{2}$ and $y / \delta$, which is caused by the fluctuation induced by the bubble forward. This energy is transferred in the form of the turbulent kinetic energy as an additional energy to overcome the pressure rise. It can be seen that at $R e_{t}=8.46 \times 10^{4}$, the turbulence ki- netic energy level near the surface is higher than that at $R e_{t}=5.08 \times 10^{4}$.

In total, the numerical results of the comparison of bubble separation profiles such as bubble length, bubble thickness, and bubble angle. In addition, information is also obtained about the comparison of the length of the massive separation of the leading edge, the thickness of the boundary layer, and the form factor. The form factor value is obtained from calculations using the numerical integration approach where the data used comes from the convergent simulation results. A summary of the numerical results for the variation $c / t=6.5$ with $R e_{t}=5.08 \times 10^{4}$ and $R e_{t}=8.46 \times 10^{4}$ as in Table 4 .

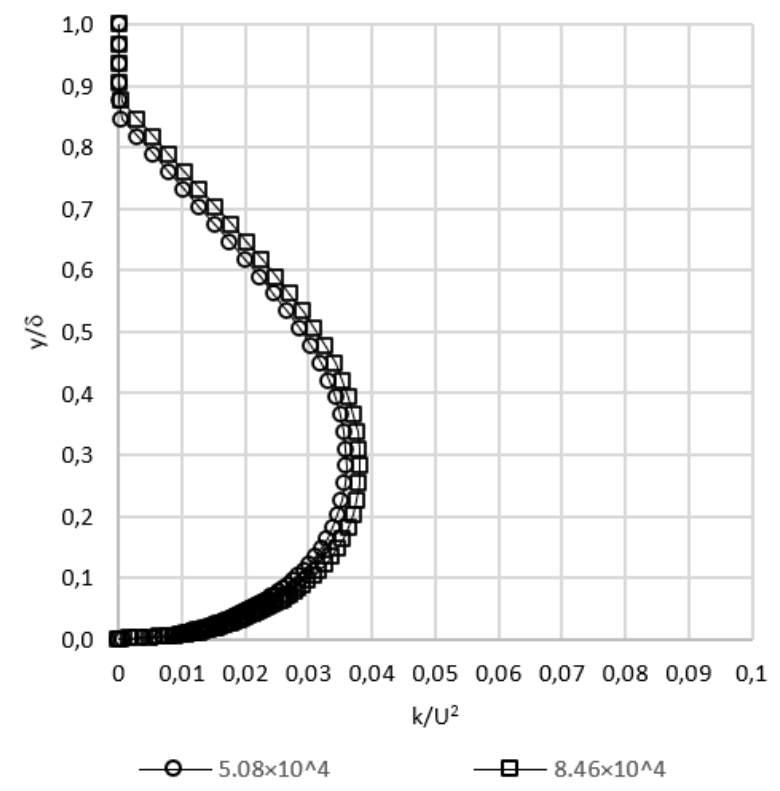

Figure 14. Plot of turbulent kinetic energy on the upper side at point $O$ 
Table 4. Analysis of separation bubble profile, massive separation length, stagnation point, and form factor

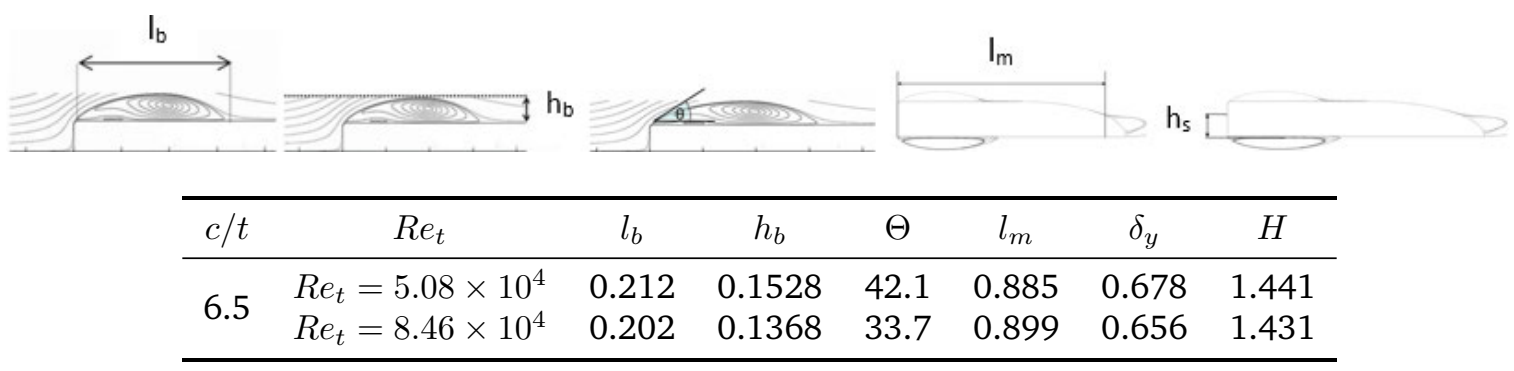

\subsection{Discussion}

In the previous section, the comparison of the flow characteristics across the circular leading edge of a thick plate was explained by displaying a comparison graph of the pressure coefficient, velocity profile and turbulent kinetic energy from the numerical results. Table 5 compares the results of all variations, namely $c / t=6.5, c / t=10$, $R e_{t}=5.08 \times 10^{4}$ and $R e_{t}=8.46 \times 10^{4}$ to analyze the separation bubble profile, massive separation length, stagnation point and form factor. It can be concluded that at the same plane length, the greater the Reynolds number, the shorter the separation bubble length so that the turbulent kinetic energy becomes larger which results in the massive separation downstream being delayed. In addition, the thickness of the bubbles formed becomes thinner as the bubbles become smaller. It is also known that the flow on the upper side is more turbulent when viewed from the form factor with the decreasing thickness of the boundary layer. Whereas at the same Reynolds number, the longer the flow support plane, the shorter the separation bubble length so that the turbulent kinetic energy is slightly larger which causes massive separation downstream to be delayed. In addition, the angle of the bubbles formed is getting smaller, but the thickness of the bubbles formed does not change significantly. It is also known that the flow on the upper side is more turbulent when viewed from the form factor with increasing thickness of the boundary layer. For all variations, the form factor value at point $O$ represents the turbulent intensity of the medium.

To support this research, the results of this study will be compared with the results of research conducted by
Rafrisah [5]. Research conducted by [5] has the same test model form as this study, for example round size $(r / t=0.1)$, variations in the length of the plane and the shape of the trailing edge. However, Rafrisah's research [5] has different Reynolds number variations from this study, namely $R e_{t}=6.76 \times 10^{4}$ and $R e_{t}=10.15 \times 10^{4}$. In the variation of $c / t=6.5$ with $R e_{t}=10.15 \times 10^{4}$, the data obtained include: length separation bubble $\left(l_{b}\right)=0.127$, separation bubble thickness $\left(h_{b}\right)=0.1448$, bubble separation angle $(\Theta)=29.5^{\circ}$, massive separation point $\left(l_{m}\right)=0.94$, and form factor $(H)=1.419$. While the variation of $c / t=10$ with $R e_{t}=10.15 \times 10^{4}$ the data obtained includes the bubble separation length $\left(l_{b}\right)=0.047$, the bubble separation thickness $\left(h_{b}\right)=0.0897$, the angle of separation bubble $(\Theta)=26.7^{\circ}$, the massive separation point $\left(l_{m}\right)=0.979$, and the form factor $(H)=1.252$. So it can be concluded that at the same rounding, the longer the flow bearing plane and the greater the Reynold number, the separation bubble profile formed and the resulting form factor value is getting smaller which results in more and more downstream separation late.

In addition, the results of this study will also be compared with the results of research conducted by Rahayu [6]. Research conducted by Rahayu [6] has the same variation as this study, namely variations in the length of the plane and the Reynolds number and the same shape of the trailing edge. However, Rahayu's study [6] has a different round size with this study, namely $r / t=0.2$. In the variation of $c / t=6.5$ with $R e_{t}=8.46 \times 10^{4}$, data is obtained in the form of bubble separation length $\left(l_{b}\right)=0.057$, bubble separation thickness $\left(h_{b}\right)=0.0321$, bubble separation angle $(\Theta)=23.6^{\circ}$, the massive point of separation $\left(l_{m}\right)=0.8966$, and the form factor $(H)=1.2845$.

Table 5. Analysis of separation bubble profile, massive separation length, stagnation point, and form factor

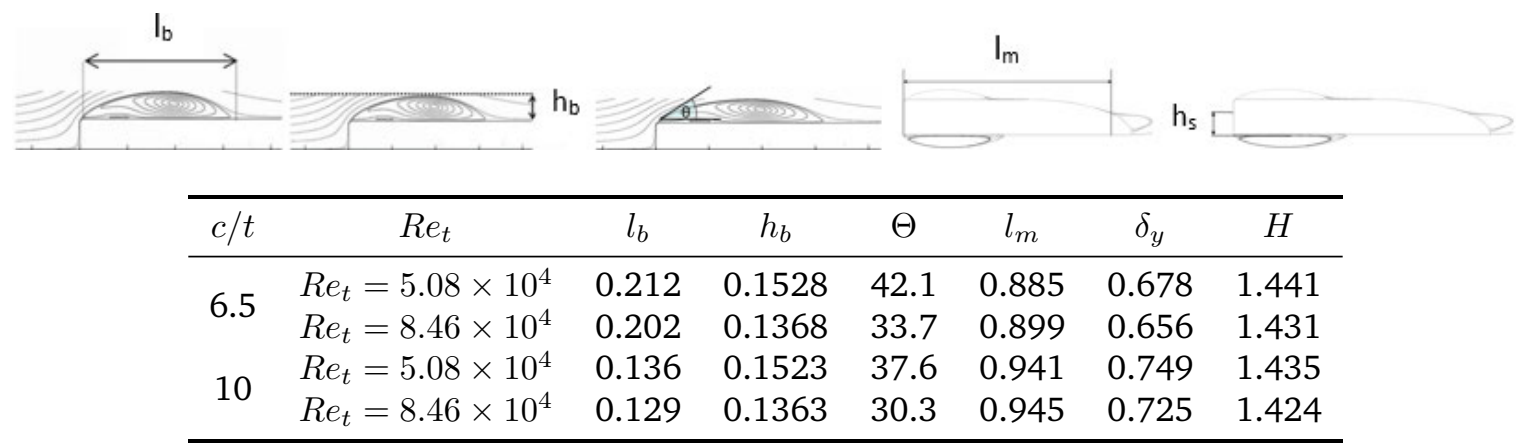


Whereas in the variation of $c / t=10$ with $R e_{t}=$ $8.46 \times 10^{4}$ the data obtained includes the length of the separation bubble $\left(l_{b}\right)=0.0631$, the thickness of the separation bubble $\left(h_{b}\right)=0.0609$, the bubble separation angle $(\Theta)=27.6^{\circ}$, the massive separation point $\left(l_{m}\right)=0.927$, and the form factor $(H)=1.274$. So it can be concluded that at the same flow bearing length and Reynolds number, the larger the rounded profile, the formation of the bubble separation profile and the smaller the resulting form factor value which results in the further delay of the downstream separation.

\section{Conclusions}

After analyzing the characteristics of the separation bubble on the edge upperside thick plate-rounded leading edge with plane length of $c / t=6.5$ and $c / t=10$ against $R e_{t}=5.08 \times 10^{4}$ and $R e_{t}=8.46 \times 10^{4}$ experimentally and numerically, the following conclusions are obtained. The longer the flow bearing plane and the greater the Reynolds number, the smaller the separation bubble profile that is formed which causes the downstream separation to be even more delayed. Overall, the most optimal variation for delaying massive separation in the downstream area is the length of the flow bearing plane $(c / t)=10$ with Reynolds number $\left(R e_{t}\right)=8.46 \times 10^{4}$.

\section{References}

[1] T. C. Corke and F. O. Thomas, "Active and passive turbulent boundary-layer drag reduction," AIAA journal, vol. 56, no. 10, pp. 3835-3847, 2018.
[2] E. Lamballais, J. Silvestrini, and S. Laizet, "Direct numerical simulation of flow separation behind a rounded leading edge: Study of curvature effects," International Journal of Heat and Fluid Flow, vol. 31, no. 3, pp. 295-306, 2010.

[3] J. Niu, Y. Wang, L. Zhang, and Y. Yuan, "Numerical analysis of aerodynamic characteristics of high-speed train with different train nose lengths," International Journal of Heat and Mass Transfer, vol. 127, pp. 188199, 2018.

[4] J. D. Anderson, Computational fluid dynamics: the basics with applications. McGraw-Hill, Inc., 1995.

[5] A. Rafrisah, Studi eksperimen dan numerik karakteristik separation bubble dari aliran dua dimensi melintasi thick-plate rounded leading edge $(r / t=0.1)$ dengan variasi Reynolds number $\left(\operatorname{Re}_{t}=6.76 \times 10^{4}\right.$ dan $\operatorname{Re}_{t}=$ $\left.10.15 \times 10^{4}\right)$ dan panjang aksial plat $(c / t=6.5$ dan $c / t=10)$. Undegraduate Thesis, Institut Teknologi Sepuluh Nopember, 2020.

[6] C. E. Rahayu, Studi eksperimen dan numerik karakteristik separation bubble dari aliran dua dimensi melintasi thick plate-rounded leading edge $(r / t=0.1)$ dengan variasi Reynolds number $\left(R e_{t}=5.08 \times 10^{4}\right.$ dan $\left.R e_{t}=8.46 \times 10^{4}\right)$ dan panjang aksial plat $(c / t=$ 6.5 dan $c / t=10$ ). Undergraduate Thesis, Institut Teknologi Sepuluh Nopember, 2020. 